\title{
Tuberculose pulmonaire en milieu carcéral au Niger : aspects épidémiologiques, diagnostiques, thérapeutiques et évolutifs
}

\author{
Kadri Sani \\ Service de Médecine Interne, Centre Hôpital Régional de Maradi, Niger \\ Harouna Amadou Mahaman Laouali \\ Service des maladies Infectieuses, Centre Hôpital Régional de Maradi, \\ Faculté des Sciences de la Santé de l'Université de Maradi, Niger
}

\section{Ada Mahaman Laminou}

Service de Médecine Interne, Hôpital National de Niamey, Niger

\section{Garba Abdoul Azize \\ Daou Mamane \\ Alberto Piubello \\ Adehossic Eric}

Service de Médecine Interne, Hôpital National de Niamey, Faculté des Sciences de la Santé, Université ABDOU M. Niamey, Niger

\section{Doi:10.19044/esj.2021.v17n14p41}

Submitted: 04 November 2020

Accepted: 23 March 2021

Published: 30 April 2021
Copyright 2021 Author(s)

Under Creative Commons BY-NC-ND

4.0 OPEN ACCESS

Cite As:

Sani K., Mahaman Laouali H.A., Laminou A.M., Azize G.A., Mamane D., Piubello A. \& Eric A. (2021). Tuberculose pulmonaire en milieu carcéral au Niger : aspects épidémiologiques, diagnostiques, thérapeutiques et évolutifs. European Scientific Journal, ESJ, 17(14), 1. https://doi.org/10.19044/esj.2021.v17n14p41

\section{Resume}

Objectif : Décrire les aspects épidémiologiques, diagnostiques, thérapeutiques et évolutifs de la tuberculose pulmonaire en milieu carcéral au Niger. Il s'agissait d'une étude transversale réalisée sur 3 ans incluant tous les cas confirmés de tuberculose pulmonaire à microscopie positive (TPM+), les co-infections $\mathrm{VIH} /$ tuberculose et les cas de tuberculose pulmonaire à microscopie négative (TPM-). Résultats : Au total 3322 étaient détenus avec une prévalence de la tuberculose de $0,84 \%$. Tous les patients étaient des hommes. La moyenne d'âge était de 36 ans avec des extrêmes allant de 21 à 62 ans. 17,85\% exerçaient dans le secteur informel.35, 71\% provenaient des milieux urbains. Sur le plan juridique $71,48 \%$ des patients étaient des 
condamnés et $36 \%$ des patients avaient une durée de détention de plus de 3 ans au moment du diagnostic. La triade faite de toux (100\% de cas) -fièvre (92,85\% de cas) -expectoration $(67,85 \%$ de cas) dominait le tableau clinique. L'amaigrissement $(46,42 \%$ de cas) et la cachexie $(42,46 \%$ de cas) constituaient l'essentiel des signes généraux. Le taux de co-infection VIH/SIDA- tuberculose s'élevait à $21,42 \%$. Sur le plan thérapeutique $82,14 \%$ des patients étaient de la catégorie $1.53,56 \%$ des patients avaient fait l'objet d'une hospitalisation tandis que 46,44\% avaient observé le traitement selon un mode ambulatoire. La guérison était de $46,42 \%$ de cas, le décès était 28,53\%. Conclusion: Des mesures préventives et thérapeutiques efficaces sont indispensables en milieu pénitencier.

Mots-clés : Tuberculose, prison, Niger

\title{
Tuberculosis in Prisons in Niger: Epidemiological, Clinical, Para-clinical, Therapeutic and Evolutionary Aspects
}

\author{
Kadri Sani \\ Service de Médecine Interne, Centre Hôpital Régional de Maradi, Niger \\ Harouna Amadou Mahaman Laouali \\ Service des maladies Infectieuses, Centre Hôpital Régional de Maradi, \\ Faculté des Sciences de la Santé de l'Université de Maradi, Niger \\ Ada Mahaman Laminou \\ Service de Médecine Interne, Hôpital National de Niamey, Niger \\ Garba Abdoul Azize \\ Daou Mamane \\ Alberto Piubello \\ Adehossic Eric \\ Service de Médecine Interne, Hôpital National de Niamey, \\ Faculté des Sciences de la Santé, Université ABDOU M. Niamey, Niger
}

\begin{abstract}
Objectives: Describe the epidemiological, diagnostic, therapeutic and evolutionary aspects of pulmonary tuberculosis in prisons in Niger. This was a cross-sectional 3-year study that included all confirmed cases of smear-positive pulmonary tuberculosis, HIV / TB co-infections, and smearnegative pulmonary tuberculosis (TPM-). Results: A total of 3322 inmates and the prevalence of tuberculosis was $0.84 \%$. All the patients were men. The average age was 36 years old with extremes ranging from 21 to 62 years old. $17.85 \%$ were in the informal sector. $3571 \%$ came from urban areas. In legal terms, $71.48 \%$ of patients were convicted and $36 \%$ of patients had a detention
\end{abstract}


period of more than 3 years at the time of diagnosis. The triad made of cough (100\% of cases) -fever ( $92.85 \%$ of cases) -expectoration $(67.85 \%$ of cases) dominated the clinical picture. Weight loss ( $46.42 \%$ of cases) and cachexia ( $42.46 \%$ of cases). The clinical signs, the bacteriological examination of the sputum as well as the x-ray (50\% of the patients) formed the basis of the diagnosis with miliary predominance. The HIV / AIDS-TB co-infection rate was $21.42 \% .82 .14 \%$ of patients were in Category $1.53 .56 \%$ of patients had been hospitalized while $46.44 \%$ had been on ambulatory therapy. The therapeutic success was $46,42 \%$ and the death rate was $28.53 \%$. Conclusion: Effective preventive and therapeutic measures are essential in a penitentiary environment.

Keywords: Tuberculosis, Prison, Niger

\section{Introduction}

La tuberculose reste encore un problème de santé publique dans le monde entier, elle continue d'être une cause majeure de mortalité. En 2018, on estime à 10 millions le nombre de nouveaux cas de tuberculose, dont 1,5 million sont morts OMS (2019).

La connaissance des facteurs de retard diagnostique permet de limiter la transmission de cette pathologie en particulier la connaissance sur le mode et les causes favorisant la contamination et le développement de la maladie, sur les signes révélateurs et sur la nécessité de faire une consultation précoce (Ravaahatra, 2017).

Les prisons peuvent favoriser la propagation de la tuberculose par le surpeuplement, une mauvaise ventilation, une mauvaise alimentation, le manque d'accès aux soins médicaux OMS, 2020).

Une grande partie des prisonniers viennent des milieux socioéconomiques défavorisés auxquels s'ajoutent d'autres difficultés dont l'accès aux soins limité, les conduites additives, les maladies mentales, l'immigration, la mauvaise alimentation, l'infections à VIH/SIDA... (OMS, 2020)

Le retard diagnostique, une mauvaise observance de traitement, la promiscuité dans les cellules, le manque d'aération des cellules ainsi que des transferts entre différents établissements pénitenciers favorisent la transmission et la propagation de la tuberculose dans les milieux carcéraux (OMS, 2020).

Au Niger, la population carcérale est estimée en 2008 à 6000 détenus répartis dans 38 établissements pénitenciers. C'est pourquoi la tuberculose doit être alors l'une des pathologies à surveiller prioritairement dans les prisons. Des moyens diagnostiques et thérapeutiques sont disponibles afin de réduire la propagation de la maladie entre détenus, aux gardiens, aux visiteurs mais aussi afin de diminuer le coût que supporte l'état dans sa prise en charge. 
Toute fois au Niger il n'existe pas d'études sur la tuberculose en milieu carcéral alors que l'efficacité de la lutte antituberculeuse doit se faire dans un cadre global intégrant toutes les composantes. Ainsi l'objectif de cette étude est de décrire les aspects épidémiologiques, diagnostiques, thérapeutiques et évolutifs de la tuberculose pulmonaire dans les maisons d'arrêt de Kollo et Niamey (Niger).

\section{Patients et Methode}

Il s'agit d'une étude rétrospective descriptive et analytique qui s'étendait sur la période allant du $1^{\text {er }}$ janvier 2008 au 31 décembre 2010 soit 3 ans.

Ce travail consistait en un recensement de tous les cas de tuberculose pulmonaire à microscopie positive $(\mathrm{TPM}+)$ ou tuberculose pulmonaire à microscopie négative (TPM-) chez les détenus à la prison civile de Niamey ou de Kollo.

Dans cette étude étaient inclus tous les cas de tuberculose pulmonaire confirmés bactériologiquement, les co-infections VIH/ tuberculose et les cas de tuberculose pulmonaire à bacilloscoipe négative suspectés cliniquement et /ou sur la base de la radiographie pulmonaire. Les détenus suivis pour une affection autre que la tuberculose n'étaient pas inclus dans cette étude.

La collecte des données était faite sur des fiches d'enquête individuelles conçues pour le besoin de l'étude à partir des dossiers médicaux des détenus permettant le recueil des informations suivantes : nom, prénom, âge, sexe, antécédents de tuberculose, notion de contage, statut VIH, fièvre, toux, expectoration, amaigrissement, sueur nocturne, dyspnée, hémoptysie, résultat de l'examen bactériologique des crachats, résultats radiologiques, l'existence d'une hospitalisation et sa durée, schéma thérapeutique et l'évolution des patients.

Le diagnostic de la tuberculose était retenu sur les arguments clinique, radiologique et bactériologique. L'examen direct des crachats était effectué par la méthode de Ziehl-Neelsen. Tous les cas de tuberculose étaient traités par des antituberculeux selon les schémas en vigueur au Niger (recommandés par l'OMS).

Pour réaliser cette étude l'autorisation de recherche a été obtenue de la faculté de médecine de l'Université Abdou Moumouni de Niamey et l'approbation du ministère de la justice.

Le traitement des données était effectué par le logiciel Excel version 12.0 et épi info version 6. 


\section{Résultats \\ Prévalence}

Durant la période de cette étude en moyenne 3322 détenus étaient gardés à la prison civile de Niamey et de Kollo.

Au total 28 cas de tuberculose étaient enregistrés dans les deux centres soit une prévalence de $0,84 \%$ (Tableau I).

Tableau $\mathbf{N}^{\circ} \mathbf{I}$ : Répartition des cas suspects et des cas confirmés de tuberculose en fonction du nombre total des détenus par année

\begin{tabular}{|c|c|c|c|}
\hline Année $\quad$ Effectifs & Effectif de détenus & Personnes suspectes & $\begin{array}{l}\text { Personnes } \\
\text { Confirmées } \\
\end{array}$ \\
\hline 2008 & 1020 & 15 & 12 \\
\hline 2009 & 1096 & 26 & 9 \\
\hline 2010 & 1208 & 10 & 7 \\
\hline TOTAL & 3324 & 51 & 28 \\
\hline
\end{tabular}

Les fréquences les plus élevées sont observées en 2008 avec 12 cas soit $42,85 \%$ et en 2009 avec $32,14 \%$ de cas.

La majorité des patients est incarcérée à la maison d'arrêt de Niamey soit $71,42 \%$ contre $28,58 \%$ à Kollo.

\section{Données sociodémographiques}

La moyenne d'âge est de 36 ans avec des extrêmes allant de 21 ans à 62 ans.

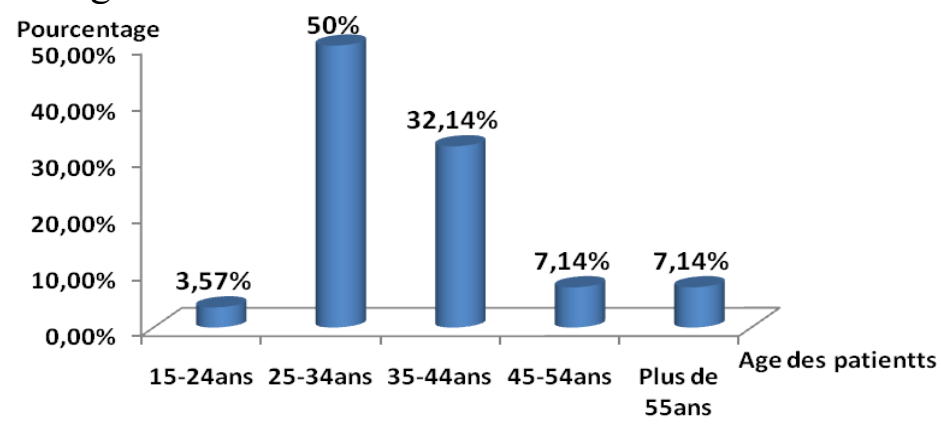

Figure 1 : répartition des patients par tranche d'âge

La plus grande fréquence a été observée dans la tranche d'âge comprise entre 25 ans et 44 ans soit $82,14 \%$ des cas et constitue la tranche d'âge la plus active de la population (Fig. 1).

L'ensemble des patients était de sexe masculin et aucune femme détenue n'était atteinte de tuberculose ; $35,71 \%$ de notre échantillon provient de centres urbains.

Une grande partie des catégories socioprofessionnelles était représentée avec $18 \%$ des patients exerçant dans le secteur informel, $14 \%$ des 
patients sont de cultivateurs et ouvriers, $14 \%$ sont des fonctionnaires et étudiants et $7 \%$ de l'échantillon prospecté sont sans emplois.

\section{Catégories des détenus}

Une prédominance des condamnés a été notée avec 20 cas soit $71,42 \%$ de notre échantillon contre $21,42 \%$ de prévenus.

Trente-quatre virgule soixante-un pourcent des patients avaient une durée de détention de plus de 3 ans au moment du diagnostic de la tuberculose.

Également 34,61\% des patients avaient une durée de détention comprise entre 3 mois à 1 an au moment du diagnostic.

Seulement 3,85\% de cette série avait un temps d'incarcération compris entre 1 mois et 3 mois lors du diagnostic de la tuberculose.

\section{Données cliniques}

Une notion de contage a été retrouvée chez $35,71 \%$ de patients. Dixsept virgule quatre-vingt-cinq pourcent de patients avaient un antécédent de tuberculose antérieure. Les antécédents de $35,71 \%$ de patients n'étaient pas précisés.

Au plan respiratoire, la toux chronique est retrouvée chez la totalité des patients soit $100 \%$ de cas suivie des expectorations chez $67,85 \%$ des patients, la dyspnée dans $28,57 \%$ de cas et de douleurs thoraciques dans $21,42 \%$ de cas.

Aucun patient n'avait présenté des signes de complications comme des hémoptysies ou de détresse respiratoire.

Concernant les signes généraux la fièvre était quasi-constante dans $92,85 \%$ de cas suivie de l'amaigrissement (sans cachexie) dans $46,42 \%$ de cas. La cachexie a été retrouvée dans $42,85 \%$ de cas. Les autres signes d'imprégnation tuberculeuse sont présents avec des faibles proportions avec respectivement $7,14 \%$ pour l'anorexie ; 3,57\% pour l'asthénie et 10,71 pour les autres signes non spécifiques.

\section{Données biologiques}

L'examen bactériologique de crachat ayant servi de base pour le diagnostic de la tuberculose, a été réalisé à l'ensemble des patients. Une prédominance de tuberculose pulmonaire à microscopie positive (TPM+) a été notée dans $85,71 \%$ de cas contre $14,28 \%$ de TPM-. Le taux de co-infection VIH/SIDA et tuberculose a été de 21,42\%.

Parmi les examens complémentaires de surveillance du traitement, seule la NFS était réalisée dans $17,85 \%$ de cas suivie de la VS dans 14,28\% de cas. Le bilan rénal a été effectué chez $10,71 \%$ des patients et le bilan hépatique a été fait chez seulement $7,14 \%$ des patients. 


\section{Données radiologiques}

L'aspect miliaire a été retrouvé dans $33,33 \%$ de cas suivi de la pleurésie dans $20 \%$ de cas et de l'infiltrat dans $20 \%$. Les cavernes ont été observées dans $13,33 \%$ de cas ; les images d'aspect normal étaient retrouvées dans $20 \%$ de cas.

\section{Données thérapeutiques}

Le traitement reposait sur les recommandations du programme national de lutte contre la tuberculose (PNLT) en vigueur au Niger au cours de la période d'étude.

Quatre-vingt-virgule quatorze pourcent des patients avaient suivi le traitement de la catégorie 1(2\{RHZE $\} / 4\{R H\})$ : deux (2) mois de quadrithérapie à base de (rifampicine, isoniazide, pyrazinamide, éthambuthol) en phase intensive initiale et quatre (4) mois de bithérapie à base de rifampicine-isoniazide en Phase de continuation. Dix-sept virgule quatrevingt-seize pourcent des patients avaient suivi le régime de traitement.

Cinquante-trois virgule cinquante-sept pourcent de l'échantillon ont fait l'objet d'une hospitalisation et $46,42 \%$ avaient suivi leur traitement en mode ambulatoire surveillé. Trente-trois pourcent des patients avaient une durée d'hospitalisation comprise entre 1 et 7 jours tandis que $20 \%$ avaient un temps d'hospitalisation supérieur à 30 jours avec des extrêmes allant de 1 à 33 jours. La durée moyenne d'hospitalisation est de 13 jours.

\section{Données évolutives}

Le taux de guérison était de $42,85 \%$. Le taux de perdu de vue a été de $17,85 \%$.Le taux de décès était de $28,57 \%$ (la cause n'était jamais précisée). Aucun cas d'échec thérapeutique ni de tuberculose résistante n'a été observé.

\section{Discussion}

Au cours de cette étude, la prévalence la tuberculose en milieu carcéral est de $0,84 \%$. Cette prévalence est variable selon les études mais reste toujours très élevée par rapport à celle de la population générale comme l'attestent les résultats des travaux réalisés en Côte d'ivoire $(0.23 \%$ vs $9.3 \%)$, au Malawi (5\%), en Ethiopie (8.33\%), en Guinée Conakry (0.075\%vs 2,4\%) (Gizachew, 2017 ; Rodrigo, 2000 ; Koffi, 1997 ; Auregan, 1995 ; Nyangulu, 1997 ; Koffi, 2017 ; Melese, 2017 ; Bah, 2012).

La durée de séjour à la prison qui vraisemblablement augmente le temps de contact avec la source de contamination, ne semble pas influencer l'importance de cette prévalence car 38,46\%des patients ont moins d'un an d'incarcération et $34,61 \%$ ont plus de 3ans d'incarcération.

L'âge moyen des patients est de 36 ans avec des extrêmes allant de 21 à 62 ans. Ces résultats sont proches de ceux de Koffi (1997) ; Nyangulu 
(1997) ; Melese (2017) ; Bah (2012) témoin de l'extrême jeunesse de la population carcérale.

La tranche d'âge comprise entre 25 ans et 44 ans $(82,14 \%)$ est la plus représentée dans cette étude, ces résultats sont semblables à ceux trouvés au Maroc par El Ghazi (2020) qui rapporte une prédominance de cette tranche d'âge qui correspond à population plus active et plus exposée à la promiscuité et aux abus.

L'ensemble des patients sont des hommes; ces résultats sont semblables à ceux de la littérature comme ceux observés par Melese (2017) ; Bah (2012) ; Adane (2018). Cela pourrait s'expliquer au fait que les maisons d'arrêt sont composées en grande partie des hommes et que la circulation des détenus entre le quartier des hommes et celui des femmes est limitée.

Contrairement aux résultats rapportés par Melese (2017); Bah (2012), Ravahatra (2017), le tableau clinique des patients est dominé par la toux ( $100 \%$ de cas), la fièvre $(92,85 \%$ de cas) et l'expectoration ( $67,85 \%$ de cas).

Dans cette série, $85,71 \%$ de patients ont tuberculose pulmonaire à microscopie positive (TPM+) contre $14,29 \%$ patients avec une tuberculose pulmonaire à microscopie négative (TPM-) dans 33,33\% de cas. Ces résultats sont proches à ceux de plusieurs séries dont Koffi (1997) ; Nyangulu (1997) ; Meles (2017) ; Bah (2012), Ravahatra (2017).

Dans cette étude, la co-infection VIH/tuberculose $(21,42 \%)$ est nettement supérieure à celle de la population générale comme rapportée dans plusieurs séries. Gizachew (2017) ; Rodrigo (2000) ; Koffi (1997) ; Nyangulu (1997); Melese (2017); Bah (2012).

A la différence de certaines études Melese (2017); Bah (2012), les signes radiologiques les plus fréquemment retrouvés dans cette série sont : l'aspect miliaire (33,33\%), suivi de la pleurésie (20\%) et de l'infiltrat (20\%).

Dans cette étude le taux de guérison est de $42,85 \%$ de cas. Ce taux de succès thérapeutique est largement en deçà du taux de guérison observé dans la population générale qui est de $79,5 \%$ en 2009 , contre un objectif de guérison de $85 \%$ fixé par l'OMS, plusieurs auteurs ont notifié un taux de guérison semblable comme Gizachew (2017); Rodrigo (2000) ; Koffi (1997); Nyangulu (1997).

Dans cette série, un taux de décès de $28,57 \%$ de cas est noté contre $5,9 \%$ observé dans la population générale en 2009. Ces résultats sont proches de ceux trouvés dans la littérature qui rapportent une proportion élevée de décès comme Koffi (1997) ; Auregan (1995) ; Noesk (2006); Bah (2012); Adane (2018). Ce taux de décès pourrait être dû au retard du diagnostic comme l'attestent la sévérité des signes généraux des patients (cachexie dans $42,85 \%$ de cas), à l'importance de la co-infection VIH/ tuberculose $(21,42 \%)$, à la malnutrition $(42,85 \%)$ et dans une certaine mesure aux problèmes d'observance et de surveillance thérapeutique. 
Dans cette étude aucun cas d'échec thérapeutique et aucun cas de tuberculose multirésistante n'est observé contrairement aux résultats de travaux de Melese (2017) ; Bah (2012) ; Adane (2018).

\section{Conclusion}

La tuberculose demeure l'une des pathologies qui posent encore de problèmes d'observance et de surveillance dans les maisons d'arrêt. Elle doit être l'une des pathologies à surveiller en priorité. Une visite médicale d'entrée, un contrôle régulier et rapproché voire un dépistage systématique doivent être institués aux regards de la forte prévalence, du taux de co-infection VIH/Tuberculose élevé et de la mortalité observée de la tuberculose en milieu carcéral au Niger. Des mesures préventives et thérapeutiques efficaces sont indispensables et un renforcement de la surveillance afin de prévenir et diminuer la morbidité et la mortalité de la tuberculose en milieu pénitencier.

\section{Conflits d'interet}

Les auteurs ne déclarent aucun conflit d'intérêt en rapport avec cet article.

\section{References:}

1. OMS (2019). Rapport mondial sur la tuberculose 2018. Document 2019 de l'Organisation mondiale de la santé ; WHO/CDS/TB/2019.15.

2. OMS (2020). Tuberculose en prison www.who.int/TB.

3. Edge CL, King EJ, Dolan K, McKee M. (2016). Prisoners co-infected with tuberculosis and HIV: a systematic review. Journal of the International AIDS Society, 19(1):20960.

4. Mrabet F.Z, M. Soualhi, R. Zahraoui, K. Marc, J. Benamor, J.E Bourkadi (2019). L'aspect de la tuberculose dans le milieu carcéral, Revue des Maladies Respiratoires, (36) : Page A250.

5. Gizachew Beza M, Hunegnaw E, Tiruneh M. (2017). Prevalence and Associated Factors of Tuberculosis in Prisons Settings of East Gojjam Zone, Northwest Ethiopia. International Journal of Bacteriology, 2017(3) : 1-7

6. El Bouazzi O (2020). Suivi Thérapeutique Pharmacologique de l'Isoniazide, European Scientific Journal, 16 (3), p 401

7. Koffi N, A.K Ngom, E. Aka-Danguy, A. Séka, N. Kouassi, D. Fadiga (1997). Tuberculose bacillifère en milieu carcéral. Notre expérience au camp pénal de Bouaké, Côte d'Ivoire. INT J TUBERC LUNG DIS11 (3): pages $250-253$.

8. CDC (1996). Prevention and control of tuberculosis in correctional facilities. . Recommendations of the Advisory Council of Elimination of tuberculosis, Vol.45/No; RR-5 
9. Roustang I., Abouzeidi A (2008). Elaboration d'une stratégie sectorielle pour la santé en milieu pénitentiaire au Niger. Rapport de mission Fév.-Mars 2008.

10. Kiter G, Arpaz S; Keskin (2003). Tuberculosis in Nazilli District Prison, Turkey, 1997-2001.INT J TUBERC LUNG DIS2 (7): 153-158.

11. Auregan G, Rakotomanana F, Ratsitorahina M, Rakotoniaina N, Rabemananjara O, Raharimana R, Boisier P (1995). Tuberculose dans les prisons à Antananarivo de 1990 à 1993. Arch Inst Pasteur Madagascar, 62 (5) : 18-23.

12. Nyangulu ds, Harries AD, Kang'ombe C, Yadidi K, Cullinan T, Maher D, Nunn P, Salaniponi FM (1997). Tuberculosis in a Prison Population in Malawi. Lancet, 350 (9087), 1284-7.

13. BANDA HT, F Gausi, A D HARRIES F M SALANIPONI (2009). Prevalence of Smear-Positive Pulmonary Tuberculosis among Prisoners in Malawi: a National Survey. Int J Tuberc Lund Dis.13 (12), p.1557-9.

14. RAO N, PAK J (2004). Prevalence of Pulmonary Tuberculosis in Karachi Central Prison. Med Assoc. 54(8), p.413-5.

15. Ravahatra Kiady, Michel Tiaray Harison, Rakotondrabe Iantsotiana Davidson, Rasoafaranirina Marie Odette, Nandimbiniaina Anjara, Rakotomizao Jocelyn Robert, Rakotoson Joelson Lovaniaina, Raharimanana Rondro Nirina (2017).Facteurs De Retard Diagnostique De La Tuberculose Pulmonaire Vus À l'Unité De Soins, De Formation Et De Recherche De Pneumologie Befelatanana ;European Scientific Journal, ESJ, 13(27), 413

16. El Ghazi I, Berni I, Menouni A, Kestemont M, Amane M, Jaafari S (2020). Profil Épidémiologique Des Pathologies Respiratoires Aux Services De Pneumologie De La Ville De Meknès (Maroc) European Scientific Journal, ESJ, 16 (33), 274

17. Noeske J, Kuaban C, Amougou G, Piubello A, Pouillet R (2006). Pulmonary Tuberculosis in the Central Prison of Douala, Cameroon East African. Medical journal, 83(1) : 25-30.

18. Séri B, Koffi A, Danel C, Ouassa T, Blehoué M-A, Ouattara E (2017). Prevalence of Pulmonary Tuberculosis Among Prison Inmates: A Cross-sectional Survey at the Correctional and Detention Facility of Abidjan, Côte d'Ivoire. PLOS ONE. 31; 12(7): e0181995.

19. Melese A, Demelash H (2017). The prevalence of Tuberculosis Among Prisoners in Ethiopia: a Systematic Review and Meta-analysis of Published Studies. Archives of Public Health, Dec Jun 12; 75(1).

20. Bah H, Cisse FA, Camara LM, Diallo OH, Diallo M, Sow OY (2012). Prévalence de la tuberculose en milieu carcéral à Conakry, République de Guinée. La Revue de Médecine Légale. Dec; 3(4):146-50. 
21. Adane K, Spigt M, Dinant G-J (2018). Tuberculosis Treatment Outcome and Predictors in Northern Ethiopian prisons: a Five-year Retrospective Analysis. BMC Pulmonary Medicine. Jun 12;18(1).

22. Samia Nassik, Aouatef Masmoudi, Salssabile Nafizy, Saad Mouine, Amine Kouisbahi, Amina Berraho (2018). Aspect Clinique Atypique De La Tuberculose Oculaire A Propos D'un Cas, European Scientific Journal, ESJ, 14(9), 122 